\title{
El análisis de la resiliencia y el rendimiento académico en los estudiantes universitarios
}

\section{Analysis of resilience and academic performance among university students}

\author{
DOI: $10.22458 /$ rna.v12i1.3534
}

Dr. Jesús Morgan Asch ${ }^{1}$

${ }^{1}$ Universidad Fidélitas, Dirección de Administración de Negocios y Economía

San José, Costa Rica; jesus.morgan.asch@gmail.com, https://orcid.org/0000-0001-8767-4427

\section{RESUMEN}

La resiliencia ha tomado un papel protagónico en diversas áreas, desde la psicológica hasta la empresarial. El objetivo es determinar la relación que guardan los factores protectores resilientes con el rendimiento académico de los estudiantes universitarios. Se aplicó el instrumento de Wagnild \& Young (1993) que logró una confiabilidad del Alpha de Cronbach de 0.91. El tamaño de la muestra fue de 497 estudiantes. Se determinó como fortalezas la confianza en sí mismo y la perseverancia. Como debilidades fueron detectadas la satisfacción personal, la ecuanimidad y el sentirse bien solo. Por tanto, se identificó que la resiliencia no explica de forma significativa el rendimiento académico.

\begin{abstract}
Resilience has taken a leading role in various areas, from the psychological to the business aspect. The objective is to determine the relationship between the resilient protective factors with the academic performance of college students. The Wagnild \& Young (1993) instrument was applied, achieving a reliability of Cronbach's Alpha of 0.91. The sample size was 497 students. Self-confidence and perseverance were determined as strengths, while the weaknesses were: personal satisfaction, equanimity and feeling well alone. it was determined that resilience does not significantly explain academic performance.
\end{abstract}

\section{RÉSUMÉ}

La résilience a pris un rôle prépondérant dans plusieurs domaines, depuis celui de la psychologie jusqu'à celui de l'entreprenariat. L'objectif est de déterminer la relation entre les facteurs de protection résilients et le rendement académique des étudiants universitaires. L'instrument de Wagnild \& Young (1993) a été appliqué en obtenant une fiabilité de l'Alpha de Cronbach de 0.91. La taille de l'échantillon a été de 497 étudiants. La confiance en soi et la persévérance ont été déterminés comme points forts. La satisfaction personnelle, l'équanimité et le fait de se sentir bien seul ont été perçus comme des points faibles. C'est pourquoi, il ressort que la résilience n'explique pas de manière significative le rendement académique.

\section{RESUMO}

A Resilience tem assumido um papel principal em várias áreas, desde a psicológica até a empresarial. O objetivo é determinar a relação que possuem os fatores de proteção resilientes com o rendimento acadêmico de estudantes universitários. Foi aplicado o instrumento de Wagnild \& Young (1993), que obteve uma confiabilidade do Alpha de Cronbach de 0,91. O tamanho da amostra foi de 497 estudantes. A autoconfiança e a perseverança foram identificadas como pontos fortes. Como fraquezas, foram detectadas a satisfação pessoal, a equanimidade e apenas se sentir bem. Portanto, identificou-se que a resiliência não explica significativamente o rendimento acadêmico.

\section{PALABRAS CLAVE: \\ RESILIENCIA, FACTORES PROTECTORES, \\ FACTORES RIESGO, ÉXITO ACADÉMICO.}

KEY WORDS:

RESILIENCE, PROTECTIVE FACTORS, RISK FACTORS, ACADEMIC SUCCESS.
MOTS CLÉS :

RÉSILIENCE, FACTEURS

DE PROTECTION,

FACTEURS DE RISQUE,

SUCCÈS ACADÉMIQUE.
PALAVRAS CHAVE: RESILIÊNCIA, FATORES DE PROTEÇÃO, FATORES DE RISCO, SUCESSO ACADÊMICO. 


\section{INTRODUCCIÓN}

La situación actual mundial se ha visto afectada por una pandemia multisistémica a raíz del virus COVID19. Ha tenido un impacto fuerte en las finanzas públicas de los diferentes países, a nivel de desempleo y economía en general. Además, la ONU (Organización de las Naciones Unidas, 2020) ha proyectado una fuerte afectación en la salud mental de las personas por diferentes razones: pérdida de seres queridos, hospitalizaciones, confinamientos, noticias y otros factores que terminan generando ansiedad, depresión, miedo e incertidumbre hacia el presente y hacia lo que depara el futuro.

Es importante considerar la brecha tecnológica existente en las sociedades como un factor que se suma a lo mencionado anteriormente, pues es generadora de ansiedad y estrés en una persona. El fenómeno de la cuarta revolución industrial (AlMulla A. , 2018) ha venido a enfrentar al individuo con tecnologías para los cuales no estaba preparado. Por ejemplo, desde el punto de vista de la educación, aprender a utilizar herramientas de aprendizaje virtual. Desde el punto de vista laboral, asumir el teletrabajo y adaptarse a herramientas tecnológicas colaborativas. En Costa Rica, esto no ha sido la excepción, la persona tiene que verse expuesta a diferentes adversidades: pandemia, nuevas tecnologías, incertidumbre, ansiedad, entre otros.

De forma específica, el estudiante universitario ha tenido que enfrentar retos adicionales. Se ha visto obligado a lidiar con múltiples factores de riesgo tales como adaptarse a tomar clases virtuales desde casa, el aislamiento con poco contacto con profesores y amigos, y el manejo de la ansiedad por su futuro (Organización de las Naciones Unidas, 2020).

El objetivo general de esta investigación es determinar la relación que guardan los factores protectores resilientes y el rendimiento académico de los estudiantes universitarios a través de un análisis cuantitativo para la recomendación de estrategias de aprendizaje complementarias en habilidades blandas.

\section{Resiliencia}

La definición de resiliencia es simple y está ampliamente aceptada, pero las que resultan más difíciles de descubrir son las condiciones que permiten iniciar un nuevo desarrollo después del trauma (Anaut, 2016). En la psicología, la resiliencia se define como "Habilidad para resurgir de la adversidad, adaptarse, recuperarse y acceder a una vida significativa y productiva" (Serna Silva et al, 2018, p. 14). Indican dichos autores que, como toda habilidad, una vez que se desarrolla a través de la práctica, esta provee la destreza para ejecutar un proceso. No se trata entonces de evitar un riesgo a toda costa, sino de afrontarlo y superarlo. Es de suma importancia trabajar la resiliencia en tiempos de rutina y no de adversidad (Gershon, 2021).

Wagnild \& Young (1993) desglosaron el constructo resiliencia en los siguientes factores de resiliencia:

a) Ecuanimidad: Denota una perspectiva balanceada de la propia vida y experiencias, tomar las cosas tranquilamente y moderando sus actitudes ante la adversidad.

b) Perseverancia: Persistencia ante la adversidad o el desaliento, tener un fuerte deseo del logro y autodisciplina.

c) Confianza en sí mismo: Habilidad para creer en sí mismo, en sus capacidades.

d) Satisfacción personal: Comprender el significado de la vida y cómo se contribuye a esta.

e) Sentirse bien solo: Nos da el significado de libertad y que somos únicos y muy importantes. (p. 8)

La resiliencia ha tenido un auge cada vez mayor en los últimos años. Su utilización ha sido reciente en el idioma español, no así en el contex to anglosajón donde es un término de uso común. Su evolución ha sido desde el individuo hacia un enfoque más comunitario y socioeducativo (Ruiz-Román, Pérez-Cea, \& Molina Cuesta, 2020). Wagnild \& Young (1993) indican que se compone de dos grandes factores: Competencia personal y la aceptación de uno mismo y la vida. Ser resiliente no es estar libre de adversidades, sino que se sobrellevarán mejor (Gershon, 2021 ).

En tiempos de la pandemia de la COVID-19, Whillans et al (2020) realizaron una investigación en 1300 estudiantes de Estados Unidos, el Reino Unido y Canadá donde se encontró que la mayoría presentaba altos grados de estrés y soledad. El $54 \%$ de la muestra reportó que de manera frecuente no sentían control sobre la situación. Indican que reflexionar sobre el significado de la vida puede aumentar la resiliencia para enfrentar la adversidad. También, mencionan los autores, que al incrementar la resiliencia se reduce el estrés personal y se produce una mayor voluntad para ayudar las familias, comunidades y al país. 
En los negocios se ha visto como a al momento de reclutar los mejores Máster en Administración de Negocios, la resiliencia es la habilidad más distintiva que se buscan entre los candidatos (Harvard Business. R y Merino Gómez, 2019).

La resiliencia, por ejemplo, es un factor trascendental en emprendimientos exitosos que, sin perseverancia ante la adversidad, no hubieran trascendido. "Se debe ser competente ante las adversidades para hablar de resiliencia, no sólo saberlas tolerar” (Dorantes, 2018).

$\mathrm{Al}$ darse a conocer de una forma más amplia el concepto, los investigadores buscan superar los límites de estudio, por eso, Harvard Business Review y Merino Gómez (2019) afirman que; "Más que la educación, la experiencia o la preparación, el nivel de resiliencia de una persona es lo que determina quién triunfa y quién fracasa" (p. 8). Esto se ve reflejado en los negocios, donde Serna Silva et al (2017) indican que estos tienen la necesidad de primero desaprender la forma de hacer y toma decisiones tradicionales, adaptarse de manera exitosa, crecer y no solo sobrevivir.

Los líderes en los negocios necesitan reevaluar su resiliencia personal y la de su equipo para lograr superar el cansancio durante tiempos de crisis (Wedell-Wedellsborg, 2020). Pues, los contratiempos son inevitables e inconvenientes por todas partes donde incluso las personas más resilientes experimentan adversidades (Ferry, 2018). Sin embargo, a pesar de dichas adversidades, Villalba Condori \& Avello Martínez (2019) encontraron, en una investigación realizada en estudiantes universitarios utilizaron el instrumento de Wagnild y Young (1993), que existe una relación altamente significativa $(\mathrm{p}<0,05)$ entre el grado de resiliencia y de satisfacción con la vida.

En el campo de la educación propiamente, la resiliencia se define como "La capacidad de recuperarse, sobreponerse y adaptarse con éxito a la adversidad, y de desarrollar competencia social, académica y vocacional pese a estar expuesto a un estrés grave o simplemente a las tensiones inherentes al mundo de hoy" (Garza Vázquez, 2016, p. 64). Indica, además, que en Latinoamérica "es un concepto relativamente nuevo, el cual ha ido adquiriendo importancia en los últimos años; los estudios sobre la temática se han enfocado principalmente hacia la educación básica y media superior; son escasas las investigaciones en la educación superior” (p. 139).

En Latinoamérica existe una diferencia entre los resultados académicos de los estudiantes según su clase social. La resiliencia permite analizar este aspecto con el fin de buscar mejores oportunidades de aprendizaje para todos, por lo que resulta clave para los sistemas educativos (Gómez \& Rivas, 2017).

Belykh (2018) afirma que la resiliencia ha permitido comprender al estudiante desde un enfoque psicosocial además, si es aplicada al ámbito educativo junto a los factores estructural y procesual se determina el cambio socioemocional que permite poder empoderarlo.

Por otra parte, se han podido establecer que los estudiantes graduados con ciertos tipos de experiencias sociales y emocionales positivas es porque son más resilientes que otros. Los que fueron competentes hicieron comunidad, se sintieron útiles, y los que tuvieron un sentido de control demostraron esa resiliencia. Se evidencia aún más esta ventaja al revisar un estudio de 2019 donde el $75 \%$ de los estudiantes universitarios dijeron que necesitan ayuda para problemas emocionales o de salud mental (Dwivedi, 2020).

\section{Rendimiento académico}

Cano Celestino y Robles Rivera (2018) describen el rendimiento académico como un fenómeno causal. Indican que es el "resultado de una serie de factores; la familia, el grupo de pares, posibilidades económicas, profesores, compañeros, motivación, interés o gusto por las actividades que desarrolla en las materias, el gusto por la carrera elegida, el desarrollo profesional, etc" (p. 8).

Según una investigación de tipo descriptivo-correlacional, realizada en 240 estudiantes de la Maestría en Gestión de los Servicios de Salud por Lora Loza (2020) en Lima, se encontró que existe una correlación positiva entre las dimensiones de la actitud (hacia las implicaciones sociales) y la motivación extrínseca con relación al rendimiento académico. Esto es reforzado por otra investigación donde se halló que los estudiantes con un rendimiento bajo presentaban dificultades en el ajuste emocional y comportamental, lo que es congruente con otros estudios previos (Sánchez-García et al 2018). Adicionalmente, Sánchez Rossel et al (2020) mencionan que, incluso la predominancia cerebral, se asocia de forma moderada con el rendimiento académico.

Igualmente, Espinosa-Castro et al (2020) determinaron que el estrés puede causar un efecto perjudicial, para ser más específico en el rendimiento académico, si no se sabe sobrellevar, igualmente reforzado por Ahmad et al (2019) quienes mostraron que el rendimiento académico tiene una correlación negativa con el estrés percibido. 
Como factores que benefician el rendimiento académico, Guzmán-Zamora et al (2020) señalan como ejemplo la adquisición de competencias y control, el interés por la materia, el engrandecimiento del yo, la valoración social y la obtención de un futuro digno, así como la habilidad de cada estudiante. De la misma forma, Paredes-Valverde (2020) indica que los estudiantes universitarios que tienen un alto grado de autoeficacia y autoconcepto, tienden a alcanzar un mayor desempeño académico.

También, Guevara Dávila et al (2019) mencionan el concepto de pensamiento crítico como un elemento favorable en la construcción del aprendizaje que a su vez favoreca el desempeño durante los estudios, así como en su ejercicio profesional. Atencia Oliva et al (2020) por su parte, indican que hallaron una correlación y valor de predicción importantes entre la dimensión de Eficacia académica del Burnout sobre rendimiento académico.

\section{Investigaciones similares en rendimiento académico y resiliencia}

Se analizaron diferentes investigaciones sobre la relación entre el rendimiento académico universitario y la resiliencia. Una de ellas se realizó en una universidad en Jalisco, México (Caldera Montes et al., 2016) donde además, se analizaron variables sociodemográficas tales como el sexo, la edad y el nivel socioeconómico. Utilizaron la Escala de Resiliencia de Wagnild y Young (1993), se obtuvieron resultados de niveles altos de resiliencia en los estudiantes universitarios y no se logró determinar diferencias significativas entre las variables carrera, sexo y edad de los estudiantes. Como recomendaciones, sugirieron seguir con la línea de investigación para diferentes muestras y carreras, además de analizar la relación de la resiliencia con otras variables.

Se realizó un estudio cuasi-experimental, cuantitativo y transversal cuyo objetivo fue determinar si los estudiantes universitarios de nuevo ingreso (Garza Vázquez, 2016) en los cuales se desarrollara la resiliencia, presentarían una menor tasa de deserción en el primer año escolar, que a quienes no se les fomentó el tema. La muestra fue de 89 estudiantes de nuevo ingreso en una Institución de Educación Superior; 41 en el grupo de tratamiento y 48 en el grupo de control. Como conclusión, se determinó que aquellos estudiantes en los cuales se les fomentaba la resiliencia presentaban una menor tasa de deserción en el primer año escolar.

Otra investigación fue centrada en la relación entre la resiliencia, el optimismo disposicional y el burnout académico. Se tomó una muestra de 463 estudiantes de la Universidad de León, y se utilizaron las escalas de Maslach Burnout Inventory-Student Survey (MBI-SS), 10-item Connor-Davidson Resilience Scale (10-item CD-RISC) y Life Orientation Test Revised (LOT-R). Se concluyó que el burnout académico presenta una relación con la resiliencia y el optimismo. En este apartado, se destaca que la resiliencia tiene una relación positiva con la eficacia.

Por otra parte, el optimismo, tal cual, también tiene efecto en la eficacia, pero no mitiga el agotamiento. Se recomendó valorar estos resultados para prevenir el burnout académico en los estudiantes universitarios (Vizoso Gómez \& Arias Gundín, 2018).

Se realizó un estudio con el objetivo de evaluar autoconcepto, valor subjetivo, expectativas y rendimiento académico. Además, analizar las relaciones entre las variables y conocer el papel que ellas desempeñan en la explicación del rendimiento académico. La muestra fue de 406 estudiantes en Colombia. Los resultados muestran que el autoconcepto, las expectativas y la edad, explicaron el rendimiento académico en matemáticas (Cárcamo et al, 2020).

Por su parte, Salvo-Garrido y otros investigadores (2020) realizaron un estudio sobre la resiliencia socioeducativa y su impacto en el rendimiento académico, para ser más específico en las matemáticas donde encontraron, a través de una regresión logística multinivel, que la confianza en sí mismo es un factor protector y como factores de riesgo la menor participación y el menor respeto a su ambiente.

Tipismana (2019) postula por medio de una investigación cuyo diseño fue transversal, comparativo y explicativo que los factores de resiliencia y afrontamiento que predicen de manera directa, indirecta y relacional el rendimiento académico son "confianza en sí mismo, capacidad social, estrategias de autofocalización emocional abierta, exposición emocional abierta y apoyo familiar social, que como predictores relevantes en expectativa de logro, aprendizaje percibido y satisfacción global. El funcionamiento familiar, es un predictor indirecto" (p. 147).

Esto podría impactar de forma negativa a la persona en el rendimiento académico, por lo que es de interés estudiar con cuáles factores de protección puede contar el estudiante para poder seguir en su carrera universitaria con un buen suceso. 


\section{METODOLOGÍA}

\section{Tipo de Estudio}

El enfoque fue cuantitativo, descriptivo, no experimental y transversal haciendo un muestreo estratificado dentro de los alumnos de la misma universidad de la carrera de Administración de Negocios se contó con el apoyo de la universidad para la recolección de datos online.

\section{Variables e Instrumentos}

La primera variable de estudio, Resiliencia, se midió a través de la aplicación del Instrumento "Escala de Resiliencia" de Wagnild \& Young (1993) que consta de 25 ítems, una escala Likert de 1 a 5, con una duración aproximada de aplicación de 5 minutos y que evalúa los factores protectores de Resiliencia de la siguiente forma:

1. Satisfacción personal: Agrupa los ítems 16, 21, 22, 25

2. Ecuanimidad: Agrupa los ítems 7, 8, 11, 12

3. Sentirse bien solo: Agrupa los ítems 5, 3, 19

4. Confianza en sí mismo: Agrupa los ítems 6, 9, 10, 13, 17, 18, 24

5. Perseverancia: $1,2,4,14,15,20$

Este instrumento se aplicó en el 2004 según Vallester (2002) en la siguiente población:

Un grupo de profesionales de la Facultad de Psicología Educativa de la UNMSM realizada en una muestra de 400 estudiantes varones y mujeres entre 13 a 18 años de tercer año de secundaria de la UGEL 03, obteniéndose una confiabilidad calculada por el método de la consistencia interna con el coeficiente alfa de Cronbach de 0.83. (p. 10)

La segunda variable del estudio es de carácter dicotómico y se basó en las calificaciones académicas obtenidas por los estudiantes. Se obtuvo el promedio de estas con el fin de categorizarlos en dos grupos: Rendimiento académico por encima del promedio y rendimiento académico por debajo del promedio.

Se revisaron las bases de datos EBSO, Google Scholar y SciELO, así como la Biblioteca virtual ELibro y de Harvard Business Review, bajo el uso de las palabras Resiliencia, Rendimiento Académico, Resiliencia Educativa y Educación en los criterios de búsqueda para publicaciones del 2016 como máxima antigüedad.

\section{Métodos Estadísticos}

\section{Procedimiento muestral}

6. Se obtuvo el consentimiento del estudiante para utilizar su información para fines investigativos, con uso de la normativa respectiva de la universidad.

7. La administración del instrumento fue individual y en línea, en los cursos correspondientes al rango que va del cuarto cuatrimestre al octavo cuatrimestre de la carrera en Administración de Negocios.

8. Se realizó la revisión de la base de datos académica para obtener el rendimiento de los estudiantes (notas de los cursos llevados durante el 2019 y el 2020) que respondieron la Escala de Resiliencia.

El universo estuvo compuesto por el total de estudiantes matriculados en la carrera de Bachillerato en Administración de Empresas. Para el tercer cuatrimestre del 2020, este número representa 1271 personas. Por tanto, se realizó un muestreo por intención, no aleatorio. El tamaño de muestra con un nivel de confianza del $95 \%$ y un margen de error del $3 \%$, fue de 497 estudiantes quienes cursaban una materia entre el cuarto cuatrimestre hasta el octavo cuatrimestre de la carrera.

Se conformó una base de datos con la información recolectada, tanto producto de la administración del instrumento de resiliencia como las calificaciones obtenidas por cada individuo. También, se utilizó el software estadístico IBM SPSS v.24. Se compararon las medianas de cada factor resiliente y del promedio general. Se analizaron y se aplicaron 
pruebas de diferencia entre medianas de acuerdo con las variables sociodemográficas: Edad y Género. No obstante, de acuerdo con las características de la muestra en cuanto a homogeneidad y a normalidad, se aplicó la diferencia entre medias a través del estadístico no paramétrico U de Mann-Whitney.

En adición, se realizó una regresión logística binaria donde se tomó como variable dependiente el promedio académico de los estudiantes, y en relación con las variables independientes, los factores de resiliencia y el nivel de resiliencia en general. Finalmente, se efectuaron las diferentes pruebas que requiere este método: Prueba de Significación de chi-cuadrado del modelo en la prueba ómnibus, cálculo de los estadísticos R-cuadrado de Cox y Snell, y R-cuadrado de Nagelkerke y se analizaron cuáles factores de resiliencia tienen incidencia significativa $(\mathrm{p}<0.01)$ en el rendimiento académico.

\section{RESULTADOS}

Se aplicó el instrumento de medición de resiliencia de Wagnild \& Young (1993) en una muestra de 497 estudiantes, este presentó una confiabilidad alta, con un Alpha de Cronbach de 0.917. Además, al realizar la prueba de normalidad Kolgomorov-Smirnov se comprobó que los datos no cumplieron con el supuesto de normalidad aplicando un nivel de significancia de $\mathrm{p}=0.05$.

Dentro de los factores de resiliencia que obtuvieron un mayor promedio destacan la confianza en sí mismo y la perseverancia. Las más débiles, y posibles factores a intervenir, la Satisfacción Personal, la Ecuanimidad y el Sentirse bien solo, tal y como se puede observar en la figura 1 que se presenta a continuación:

\section{Figura 1}

Comparación de medición de factores de resiliencia de los estudiantes

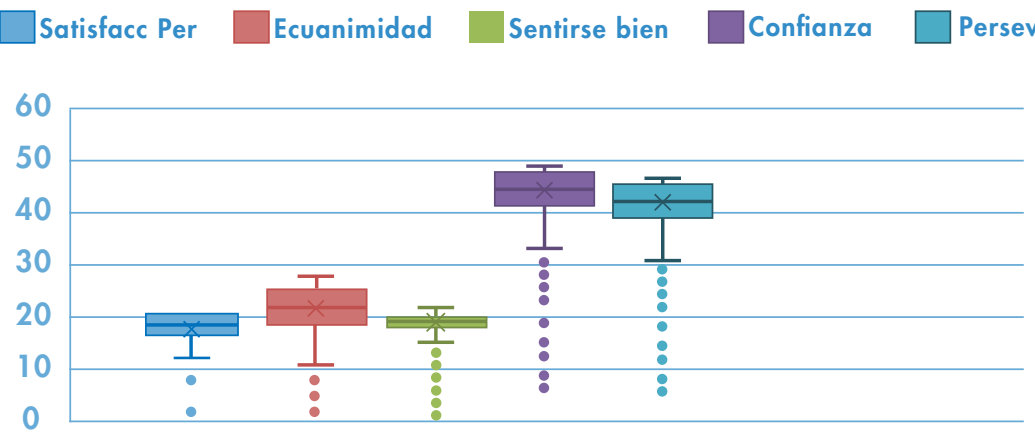

Fuente: elaboración propia. 
Se realizó la prueba no paramétrica de U de Mann-Whitney y se comprobó que no existe una diferencia estadísticamente significativa en los diferentes factores de resiliencia entre hombres y mujeres $(\mathrm{p}=0.05)$ ni por edad $(\mathrm{p}=0.05)$, tal y como se muestra en la tabla $1:$

\section{Tabla 1}

Análisis de diferencias en los factores de resiliencia según el género

Resumen de contraste de hipótesis

\begin{tabular}{|c|c|c|c|c|}
\hline & Hipótesis nula & Prueba & Sig $a b$ & Decisión \\
\hline 1 & $\begin{array}{l}\text { Las medidas de Satis Personal son las } \\
\text { mismas entre categorías de Genero }\end{array}$ & $\begin{array}{l}\text { Prueba de la mediana para muestras } \\
\text { independientes }\end{array}$ & $496^{\circ}$ & Conserve la hipótesis nula \\
\hline 2 & $\begin{array}{l}\text { Las medianas de Ecuanimidad son las } \\
\text { mismas entre las categorías de Género }\end{array}$ & $\begin{array}{l}\text { Prueba de la mediana para muestras } \\
\text { independientes }\end{array}$ &, $378^{\circ}$ & Conserve la hipótesis nula \\
\hline 3 & $\begin{array}{l}\text { Las medianas de Sentirse bien solo son } \\
\text { las mismas entre categorías de Género }\end{array}$ & $\begin{array}{l}\text { Prueba de la mediana para muestras } \\
\text { independientes }\end{array}$ &, $500^{\circ}$ & Conserve la hipótesis nula \\
\hline 4 & $\begin{array}{l}\text { Las medianas de Confianza en si mismo } \\
\text { son las mismas entre categorías de } \\
\text { Género }\end{array}$ & $\begin{array}{l}\text { Prueba de la mediana para muestras } \\
\text { independientes }\end{array}$ &, $161^{\circ}$ & Conserve la hipótesis nula \\
\hline 5 & $\begin{array}{l}\text { Las medianas de Perseverencia son las } \\
\text { mismas entre las categorías de Género }\end{array}$ & $\begin{array}{l}\text { Prueba de la mediana para muestras } \\
\text { independientes }\end{array}$ &, $995^{\circ}$ & Conserve la hipótesis nula \\
\hline 6 & $\begin{array}{l}\text { Las medianas de PromGeneral son las } \\
\text { mismas entre categorías de Género }\end{array}$ & $\begin{array}{l}\text { Prueba de la mediana para muestras } \\
\text { independientes }\end{array}$ &, $316^{\circ}$ & Conserve la hipótesis nula \\
\hline
\end{tabular}

a. El nivel de significación es de, 050 .

b. Se muestra la significancia asintótica.

c. Significación asintótica corregida de continuidad de Yates.

Fuente: elaboración propia.

Para determinar si la resiliencia influye en el rendimiento académico desde un enfoque estadístico, se aplicó la Regresión Logística Binaria, categorizando la variable rendimiento como una variable dicotómica (Rendimiento por encima del promedio y rendimiento por debajo del promedio). En la tabla 2 se muestra el nivel de significación de chi-cuadrado en la prueba ómnibus y, al ser la significación del modelo mayor a $\mathrm{p}=0.05$, se concluye que este no ayuda a explicar el evento. Es decir, las variables independientes (factores de resiliencia) no explican la variable dependiente (rendimiento académico). 


\section{Tabla 2}

Significación de chi-cuadrado del modelo en la prueba ómnibu

Prueba de ómnibus

\begin{tabular}{l|l|c|c|l}
\hline \multicolumn{2}{l|}{} & Chi-cuadrado & gl & Sig \\
\hline \multirow{2}{*}{ Paso 1 } & Paso & 4,329 & 5 & Conserve la hipótesis nula \\
\cline { 2 - 5 } & Bloque & 4,329 & 5 & Conserve la hipótesis nula \\
\cline { 2 - 5 } & Modelo & 4,329 & 5 & Conserve la hipótesis nula \\
\hline
\end{tabular}

Fuente: elaboración propia.

Como se puede apreciar en la tabla 3 , el modelo (factores de resiliencia) no es significativo, explica entre el 0.009 y el 0,012 de la variable rendimiento académico.

\section{Tabla 3}

R-cuadrado de Cox y Snell, y R-cuadrado de Nagelkerke

Resumen del modelo

\begin{tabular}{c|l|c|c}
\hline Paso & logaritmo de la verosimilitud -2 & R cuadrado de Cox y Snell & R cuadrado de Nagelkerke \\
\hline 1 & $677,154^{a}$ &, 009 &, 012 \\
\cline { 2 - 4 }
\end{tabular}

a. La estimación ha terminado en el número de iteración 3 porque las estimaciones de parámetro han cambiado en menos de, 001

Fuente: elaboración propia.

En la tabla 4 se concluye que ninguno de los factores de resiliencia explica de forma significativa $(\mathrm{p}=0.05)$ el Rendimiento Académico Universitario de los estudiantes.

\section{Tabla 4}

Variables en la Ecuación

Variables en la ecuación

\begin{tabular}{|c|c|c|c|c|c|c|c|}
\hline & & B & $\begin{array}{l}\text { Error } \\
\text { estándar }\end{array}$ & Wald & gl & Sig & $\operatorname{Exp}(B)$ \\
\hline \multirow[t]{6}{*}{ Paso $1^{a}$} & SatisPersonal &,- 156 &, 138 & 1,277 & 1 &, 258 &, 855 \\
\hline & Ecuanimidad &,- 138 &, 112 & 1,506 & 1 & 220 & 871 \\
\hline & Sentirsebiensolo &, 092 & 145 &, 400 & 1 &, 527 & 1,096 \\
\hline & Confianzaensimismo &, 233 & 206 & 1,168 & 1 & ,280 & 1,249 \\
\hline & Perseverancia &, 030 & ,203 &, 022 & 1 &, 882 & 1,031 \\
\hline & Constante &,- 207 & ,708 & 085 & 1 & ,770 & 813 \\
\hline
\end{tabular}

a. Variables especificadas en el paso 1: SatisPersonal, Ecuanimidad, Sentirsebiensolo, Confianzaensimismo, Perseverancia 


\section{DISCUSIÓN DE RESULTADOS}

El instrumento aplicado para medir la resiliencia (Wagnild \& Young , 1993) es confiable $(\boldsymbol{\alpha}=0.917)$, lo que demuestra la consistencia interna de la prueba que indica la interrelación entre los distintos ítems del instrumento aplicado y, en este sentido, separa del conjunto la variación que corresponde a factores comunes de los ítems y la que corresponde a factores únicos de cada uno de ellos, esto puede tomarse como una forma de estimación de la equivalencia de los componentes entre sí (Cervantes, 2015).

Los factores de resiliencia: Confianza en sí mismo y la perseverancia, son fortalezas dentro del grupo bajo análisis. Esto hace más probable que el estudiante sobrepase la adversidad sin menoscabo de su propio desarrollo académico (Garza Vázquez, 2016) lo cual sugiere un derrotero a la universidad para tomar acciones en aras de fortalecer aún más estos aspectos.

Los otros factores protectores de resiliencia señalan mayores oportunidades de mejora por el bajo nivel encontrado. Estos son: la satisfacción personal, el sentirse bien solo y la ecuanimidad. En ese sentido, Kochetkova et al (2019) indican que el bienestar psicológico afecta el desempeño de los estudiantes esto, además, se ve reforzado con lo señalado por Flores Ortiz \& Sánchez Cancino (2016):

Los aspectos biológicos y sociales son muy importantes para el rendimiento académico, pero evidentemente los psicológicos también son centrales. Esta área ha explorado factores como la inteligencia, personalidad, autoestima, auto concepto, toma de decisiones, resistencia a la frustración, entre los más relevantes que, independientemente de la edad o grado que cursa el estudiante influye en el rendimiento académico. (p. 89)

Los datos demuestran cómo el nivel de resiliencia es similar entre hombres y mujeres. Esto es coincidente con investigaciones similares donde tampoco se encontró una diferencia estadística significativa en variables sociodemográficas como carrera, sexo y edad de los estudiantes (Caldera Montes et al, 2016). Por tanto, puede servir a la universidad como base para impulsar aún más la promoción de mujeres en ciencias empresariales.

Sobre la variable rendimiento académico, esta puede verse afectada por muchas otras diferentes de los factores personales, tales como entorno familiar, formación previa, situación económica o laboral y muchas otras. Así lo señalan también Cano Celestino y Robles Rivera (2018), por lo que se podría prever, para darle continuidad a esta investigación, incorporar mayor cantidad de variables demográficas tales como: el clima familiar, situación económica, profesores, compañeros, motivación, interés o gusto por las actividades que desarrolla en las materias, el gusto por la carrera elegida, el desarrollo profesional y así, tener un concepto más integral del rendimiento académico.

A pesar de que la hipótesis que se plantea inicialmente no se pudo comprobar, esta no siempre tiene que resultar ser verdadera (Espinoza, 2018). Menciona dicho autor que el éxito que pueda tener dicha hipótesis para explicar la realidad será siempre coyuntural, sujeta a correcciones o a sustitución. Si bien es cierto, de forma cuantitativa no se logró constatar la sospecha inicial del investigador, se aporta evidencia cualitativa que sugiere la relación entre la resiliencia y el rendimiento académico, por lo que hay referencias que sugieren que se pueda perfeccionar la hipótesis. En ese sentido, sería factible utilizar otro instrumento con mayor especificidad y más reciente, para medir la resiliencia académica y así obtener un resultado más apegado a la realidad del ambiente académico. 


\section{CONCLUSIONES}

La resiliencia es la capacidad, a nivel personal u organizacional, de anticipar, resistir, recuperarse y evolucionar al enfrentar una adversidad. A través de la revisión de literatura, se evidencia cómo es de ayuda el superarse a nivel personal y profesional. El rendimiento académico es el resultado de una serie de variables ambientales y personales que pueden afectar si un estudiante finalmente tiene un promedio alto o no. Las diferentes investigaciones previas que abarcan aspectos aislados de la resiliencia han encontrado una influencia positiva en el rendimiento académico.

A partir de los resultados y la revisión de los dos grandes conceptos abordados en este artículo, se identifican ciertos elementos de estudios en el área académica que podrían sugerir una relación positiva entre la resiliencia y el rendimiento académico. Sin embargo, a nivel estadístico cuantitativo, esta no pudo ser comprobada. Es posible que el instrumento para medir resiliencia utilizado en este estudio se concentra de forma genérica en habilidades para la vida, de acuerdo con los diferentes factores resilientes que lo componen. Es por esto que sería recomendable utilizar otro instrumento de medición más específico y especializado en evaluar la resiliencia académica; por ejemplo, el cuestionario de resiliencia para los estudiantes universitarios CRE-U (Peralta et al, 2006).

- De la muestra analizada $(n=497)$, se encontró que no hay diferencias estadísticamente significativas en los niveles de resiliencia general y en sus factores entre los diferentes grupos sociodemográficos, en este caso, segmentados por género y por edad.

- Así mismo, en dicha muestra, se destacaron los factores de resiliencia Confianza en sí mismo y Perseverancia como las fortalezas que presentan los estudiantes universitarios, y como debilidades, la Satisfacción Personal y Sentirse bien. No se presentaron diferencias significativas en cuanto a género y edad en los niveles de resiliencia.

- Se determinó que no existen diferencias estadísticamente significativas de los factores resilientes entre los estudiantes con Rendimiento Académico por encima del promedio y estudiantes con Rendimiento Académico por debajo del promedio.

Se recomienda, como una línea de investigación futura, profundizar con un instrumento específico para resiliencia académica (CRE-U) en cómo las habilidades resilientes pueden asociarse al rendimiento académico y estas puedan servir a su vez, para mejorar lo a los estudiantes universitarios, a nivel cuantitativo y con muestras de mayor tamaño. De esta manera, establecer un posible programa de reforzamiento de resiliencia a nivel del estudiantado para mejorar en los estudiantes los factores resilientes: satisfacción personal, ecuanimidad y sentirse bien solo, mediante un refuerzo más hacia el enfoque integral de la educación superior. 


\section{REFERENCIAS}

Ahmad, D., Ahmad Baig, I., \& Munir, N. (2019). Relación del rendimiento académico con el estrés percibido y el índice de masa corporal. Revista Dilemas Contemporáneos: Educación, Política y Valores(57), 1-17. http://www. dilemascontemporaneoseducacionpoliticayvalores.com/

AlMulla, A. (2018). 5 pasos para desarrollar resiliencia emocional en el trabajo. Foro Económico Mundial. https://www. weforum.org/es/agenda/2018/02/5-pasos-para-desarrollar-resiliencia-emocional-en-el-trabajo

Anaut, M. (2016). ¿¿Por qué la resiliencia? Editorial Gedisa. https://elibro.net/es/lc/ufidelitas/titulos/61226

Atencia Oliva, D., Plaza Gómez, M. T., \& Hernández Riaño, H. E. (2020). Resiliencia, burnout y fracaso académico en estudiantes de Ingeniería de la Universidad de Córdoba,. Revista Espacios, 41(11), 23-38.

Belykh, A. (2018). Resiliencia e inteligencia emocional conceptos complementarios para empoderar al estudiante. Revista Latinoamericana De Estudios Educativos, 48(1), 255-282. https://doi.org/10.48102/rlee.2018.48.1.81

Business Review, H., \& Merino Gómez, B. (2019). Resiliencia. Editorial Reverté.

Caldera Montes, J. F., Aceves Lupercio2, B. I., \& Reynoso González, Ó. U. (2016). Resiliencia en estudiantes universitarios. Un estudio comparado entre carreras. Psicogente, 19(36), 227-239. http://doi.org/10.17081/psico.19.36.1294

Cano Celestino, M. A., \& Robles Rivera, R. (2018). Factores asociados al rendimiento académico en estudiantes. Revista Mexicana de Orientación Educativa, 15(35), 1-25. https://doi.org/10.31206/rmdo072018

Cárcamo, C., Moreno, A., \& del Barrio, C. (2020). Diferencias de género en matemáticas y lengua: rendimiento académico, autoconcepto y expectativas. SUMA PSICOLÓGICA, 27(1), 27-34. https://doi.org/10.14349/sumapsi.2020.v27.n1.4

Cervantes, V. H. (2015) Interpretaciones del coeficiente alpha de cronbach. Revista Avances en Medición 3, 9-28

Dorantes, R. (22 de Febrero de 2018). Qué es la resiliencia y por qué la necesitas para ganar. www.entrepreneur.com: https://getpocket.com/a/read/2085361167

Dwivedi , P. D. (2020). How to Build Resilience During College-When it Matters Most. Harvard Business Review. https://hbr.org/2020/05/how-to-build-resilience-during-college-when-it-matters-most

Espinosa-Castro, J.-F., Hernández-Lalinde, J., Rodríguez, J. E., Chacín, M., \& Bermúdez-Pirela, V. (2020). Influencia del estrés sobre el rendimiento académico. Revista AVFT, 39(1), 63-69.

Espino Freire, E.E. (2018). La hipótesis en la investigación. Revista Mendive 16(1)

Ferry, K. (2018). For your improvement. Korn Ferry Leadesership Arquitect.

Flores Ortiz, M. E., \& Sánchez Cancino, F. (2016). El bajo rendimiento académico: la mirada de los alumnos. Grupo Editorial Éxodo. https://elibro.net/es/lc/ufidelitas/titulos/130384

Garza Vázquez, L. H. (2016). Resiliencia en la Educación Superior para prevenir la deserción escolar en el primer año: caso Unidad Académica Multidisciplinaria de Ciencias, Educación y Humanidades. Universidad Autónoma de Tamaulipas.

Gershon, N. (2021) Centro de Resiliencia Cohen. Conferencia Principios de Resilencia en el sistema educativo. Israel

Gómez, G., \& Rivas, M. (Diciembre de 2017). Resiliencia académica, nuevas perspectivas de interpretación del aprendizaje en contextos de vulnerabilidad social. Calidad en la educación(47), 215-233. http://dx.doi.org/10.4067/So71845652017000200215

Guevara Dávila, F., Pérez Moreano, Y., \& Macazana Fernández, D. (2019). Pensamiento crítico y su relación con el rendimiento académicoenlainvestigación formativa delos estudiantes universitarios. Revista Dilemas Contemporáneos: Educación, Política y Valores(13), 1-17. http://www.dilemascontemporaneoseducacionpoliticayvalores.com/

Guzmán-Zamora, N., \& Gutiérrez-García, R. A. (2020). Motivación escolar: metas académicas, estilos atribucionales y rendimiento académico en estudiantes de educación media. Revista AVFT , 290-298.

Harvard Business. R y Merino Gómez, B. (2019). Resiliencia. Editorial Reverté. 
Kochetkova, G., Prodanova, N., Akifev, I., Seluch, M., \& Murtazina, D. (2019). Interdependencia del bienestar psicológico y el rendimiento académico de los estudiantes de ingeniería. Revista Dilemas Contemporáneos: Educación, Política y Valores, 1-19. http://www.dilemascontemporaneoseducacionpoliticayvalores.com/

Lora Loza, M. G. (2020). Actitud, motivación y ansiedad y su relación con el rendimiento académico en estudiantes del Posgrado. Universidad César Vallejo 2017. , 20(1), 37-44. http://dx.doi.org/10.24265/horizmed.2020.v20n1.06

Marston, A., \& Marston, S. (19 de Febrero de 2018). To Handle Increased Stress, Build Your Resilience. Recuperado el 23 de Febrero de 2018, de Harvard Business Review: https://hbr.org/2018/02/to-handle-increased-stress-buildyour-resilience

Moraleda, A. (7 de Febrero de 2018). Cómo motivar al trabajador en tiempos de inseguridad laboral. Retina El País. Recuperado el 23 de Febrero de 2018, de https://retina.elpais.com/retina/2018/02/06/talento/1517912850_105952.html

Ordoñez Chillarón, E. (27 de Setiembre de 2017). La inteligencia emocional será la base del éxito laboral en el futuro. Yorokobu. Recuperado el 16 de Febrero de 2018, de http://www.yorokobu.es/inteligencia-emocional-y-trabajo-futuro/

Organización de las Naciones Unidas. (10 de Octubre de 2020). Noticias ONU https://news.un.org/es/ story/2020/10/1482212

Paredes-Valverde, Y., Quispe-Herrera, R., \& Garate-Quispe, J. (2020). Relaciones entre autoeficacia, autoconcepto y rendimiento académico en estudiantes universitarios de la Amazonía peruana. Revista Espacios, 41(18), 18-24.

Peralta Díaz, S., Ramírez Giraldo, A., Castaño Buitrago, H. (2006). Factores resilientes asociados al rendimiento académico en estudiantes pertenecientes a la Universidad de Sucre (Colombia). Psicología desde el Caribe. 17, 196-219.

Ruiz-Román, C., Pérez-Cea, J. J., \& Molina Cuesta, L. (2020). Evolución y nuevas perspectivas del concepto de resiliencia de lo individual a los contextos y a las relaciones socioeducativas. Educatio Siglo XXI, 38(2), 213-232. http:// revistas.um.es/educatio

Salvo-Garrido, S., Miranda Vargas, H., Vivallo Urra, O., Gálvez-Nieto, J. L., \& Miranda-Zapata, E. (2020). Estudiantes Resilientes en el Área de Matemática: Examinando los Factores Protectores y de Riesgo en un País Emergente. Revista Iberoamericana de Diagnóstico y Evaluación, 2(55), 43-57. https://doi.org/10.2 1865/RIDEP55.2.04

Sánchez Rossel, M. C., Gómez-Arteta, I. I., \& Bonifaz Valdez, B. (2020). Predominio cerebral y rendimiento académico en los estudiantes de la facultad de educación de la Universidad Nacional del Altiplano (UNA)-Puno. Revista de Investigación en Comunicación y Desarrollo, 11(2), 97-106. https://doi.org/10.33595/2226-1478.1 1.2.432

Sánchez-García, M. d., Lucas-Molina, B., Fonseca-Pedrero, E., Pérez-Albéniz, A., \& Paino, M. (2018). Emotional and behavioral difficulties in adolescence: Relationship with emotional well-being, affect, and academic performance. Annals of psychology, 34(3), 482-489. http://dx.doi.orc\%7D/10.6018/analesps.34.3.296631

Serna Silva, G., Zenozain Cordero, C., \& Schmidt Urdanivia, U. (2017). La resiliencia: un factor decisivo para el crecimiento y mejora de las organizaciones. Gestión en el Tercer Milenio, 20(39), 13-24.

Tipismana, O. (2019). Factores de Resiliencia y Afrontamiento como Predictores del Rendimiento Académico de los Estudiantes en Universidades Privadas. Revista Iberoamericana sobre Calidad, Eficacia y Cambio en Educación, 17(2), 147-185. https://doi.org/10.15366/reice2019.17.2.008

Vallester, L. (2002). Escala de Resiliencia (ER).

Villalba Condori, K., \& Avello Martínez, R. (2019). Resilience as a factor determining satisfaction with life among university students. Educación Médica Superior, 33(3), 1-15.

Wagnild, G., \& Young, H. (1993). Escala de Resiliencia (ER). Journal of Nursing Measurement, 1(2), 165-178.

Wedell-Wedellsborg, M. (15 de Diciembre de 2020). How to Lead When Your Team Is Exhausted — and You Are, Too. Harvard Business Review. https://hbr.org/2020/12/how-to-lead-when-your-team-is-exhausted-and-you-are-too

Whillans, A., Giurge, L. M., Macchia, L., \& Yemiscigil, A. (2020). Why a Covid-19 World Feels Both Tiring and Hopeful for College Students. Harvard Business Review, https://hbr.org/2020/08/why-a-covid-19-world-feelsboth-tiring-and-hopeful-for-college-students. 